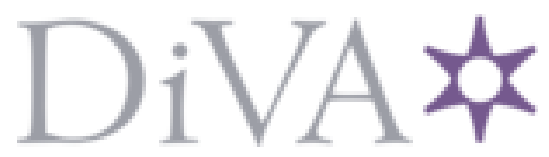

http://www.diva-portal.org

\title{
Preprint
}

This is the submitted version of a paper published in Chemistry and Biodiversity.

Citation for the original published paper (version of record):

Andersson, C., Berthold, C., Högbom, M. (2012)

A dynamic c terminal segment in the mycobacterium tuberculosis $\mathrm{mn} / \mathrm{fe} \mathrm{r} 2 \mathrm{lox}$ protein can adopt a helical structure with possible functional consequences.

Chemistry and Biodiversity, 9(9): 1981-1988

http://dx.doi.org/10.1002/cbdv.201100428

Access to the published version may require subscription.

N.B. When citing this work, cite the original published paper.

Permanent link to this version:

http://urn.kb.se/resolve?urn=urn:nbn:se:su:diva- 81820 
Chem Biodivers. 2012 Sep;9(9):1981-8. doi: 10.1002/cbdv.201100428.

\section{A dynamic C-terminal segment in the Mycobacterium tuberculosis Mn/Fe R2lox protein can adopt a helical structure with possible functional consequences.}

Andersson CS, Berthold CL, Högbom M.

Stockholm Center for Biomembrane Research, Department of Biochemistry and Biophysics, Arrhenius Laboratories for Natural Sciences, Stockholm University, SE-10691 Stockholm. 


\section{A Dynamic C-terminal Segment in the Mycobacterium tuberculosis $\mathrm{Mn} / \mathrm{Fe}$ R2lox Protein can Assume a Helical Structure with Possible Functional Consequences}

Charlotta S. Andersson, Catrine L. Berthold, and Martin Högbom*

Stockholm Center for Biomembrane Research, Department of Biochemistry and Biophysics, Arrhenius Laboratories for Natural Sciences, Stockholm University, SE-10691 Stockholm, Sweden

(phone: +46 81621 10; fax: +46 81536 79; e-mail: hogbom@dbb.su.se) 


\begin{abstract}
Mycobacterium tuberculosis R2-like ligand binding oxidase (MtR2lox) belongs to a recently discovered group of proteins that are homologous to the ribonucleotide reductase R2 proteins. MtR2lox carries a heterodinuclear $\mathrm{Mn} / \mathrm{Fe}$ cofactor and, unlike R2 proteins, a large ligand-binding cavity. A unique tyrosine-valine crosslink is also found in the vicinity of the active site. To date, all known structures of R2 and R2lox proteins show a disordered C-terminal segment. Here, we present two new crystal forms of MtR2lox, revealing an ordered helical C-terminal. The ability of alternating between an ordered and disordered state agrees well with bioinformatic analysis of the protein sequence. Interestingly, ordering of the C-terminal helix shields a large positively charged patch on the protein surface, potentially used for interaction with other cellular components. We hypothesize that the dynamic C-terminal segment may be involved in control of protein function in vivo.
\end{abstract}

Keywords: Structural dynamics, Bioinorganic, ribonucleotide reductase, heterodinuclear, oxidase 


\section{Introduction}

$\mathrm{R} 2$ like ligand binding oxidases (R2lox) is a recently discovered group of proteins that is homologous in sequence and structure to the radical generating ribonucleotide reductase subunit R2 [1,2]. The structure of Mycobacterium tuberculosis $\mathrm{R} 210 x$ (MtR2lox) has been determined and contains a heterodinuclear $\mathrm{Mn} / \mathrm{Fe}$ cofactor. Unlike the $\mathrm{R} 2$ proteins, $M t \mathrm{R} 2$ lox has a large ligand-binding cavity directly accessing the dinuclear metal site and a unique tyrosine-valine covalent crosslink in the vicinity of the active site [1]. The in vivo function for the R2lox group is still unknown but the structural features of the protein and the properties of the heterodinuclear cofactor suggests that they may be involved in challenging chemical oxidations of hydrophobic substrates. Ribonucleotide reductase R2 subunits, on the other hand, use a dinuclear cofactor (di-iron, di-manganese or heterodinuclear $\mathrm{Mn} / \mathrm{Fe}$ ) to generate and store an essential radical that is reversibly delivered to the catalytic R1 subunit [3-6]. R2 proteins from all subgroups share a disordered C-terminus, and determined crystal structures all lack approximately 30 residues that are not visible in the electron density. The C-terminal segment of protein $\mathrm{R} 2$ is known to be involved in binding and radical delivery to the $\mathrm{R} 1$ subunit and the disorder propensity of this segment is thus a functional feature [5]. In the, to date, only solved structure of an R2lox protein, $M t \mathrm{R} 2 \mathrm{lox}$, the C-terminal 24 residues are also disordered [1] but the structural consequences and functional reason, if any, remain unclear.

Here, we present crystal structures of $M t \mathrm{R} 2$ lox in two new space groups revealing an ordered helical C-terminal segment. The C-terminal helix is involved in crystal packing mediated by a metal ion from the crystallization 
solution, something that may have enabled crystallographic capture of the ordered state. Interestingly, the ordered C-terminal segment packs against a concave positively charged patch on the protein and efficiently modulates the surface properties. This type of patch may be used for protein-protein, or proteinmembrane interactions [7-11] and we hypothesize that the dynamic C-terminal segment may function to control such features by physically and electrostatically shielding the putative interaction surface.

\section{Experimental Section}

\section{Protein Preparation}

R2lox (Rv0233) from M. tuberculosis H37Rv was produced in Escherichia coli BL21(DE3) and purified as previously described [1]. The bacteria were grown in Luria-Bertani media either with or without the supplement of $2 \mathrm{mM} \mathrm{MnCl}_{2}$. In short, the histidine-tagged protein was purified by gravity flow on Ni-NTA agarose followed by size-exclusion chromatography on a Hiload 16/60 Superdex 200 column (GE Healthcare). The purified protein, in $50 \mathrm{~mm}$ 2-amino-2(hydroxymethyl)propane-1,3-diol (Tris) $\mathrm{HCl}, \mathrm{pH} 7.5,150 \mathrm{~mm} \mathrm{NaCl}$, and 5\% glycerol, was concentrated to $30 \mathrm{mg} / \mathrm{ml}$ and stored in $-80^{\circ} \mathrm{C}$ after flash cooling in liquid nitrogen. Metal content in the protein samples were analyzed as described previously [1]. The protein was diluted to $10 \mathrm{mg} / \mathrm{ml}$ prior to crystallization setup. 
Crystallization trials were performed in room temperature by the sitting drop vapor diffusion technique with previously established conditions [1]. The previously reported crystal form with spacegroup $\mathrm{P} 3_{2} 21$, contained one monomer per asymmetric unit and diffracted to $1.9 \AA$ A resolution [1]. Two new crystal forms were produced, both with a reservoir solution containing $180 \mathrm{mM}$ calcium acetate, $15-18 \%$ polyethylene glycol 18000 , and $0.1 \mathrm{M}$ sodium cacodylate $\mathrm{pH}$ 6.5-6.8. The first crystal form grew in space group $\mathrm{C} 222_{1}$ and a native data set was collected at $\lambda=1.127 \AA$, to a resolution of $2.75 \AA$. The second crystal form belonged to space group $\mathrm{P} 22_{1} 2_{1}$, and two anomalous data sets used for metal identification were collected, one at the high energy side of the $K$-edge for iron $(\lambda=1.7 \AA)$ and one at the high-energy side of the $K$-edge for manganese $(\lambda=1.8$ $\AA ̊$ ). All X-ray diffraction data were collected at beamline ID29 at the European Synchrotron Radiation Facility, Grenoble, France. Data were collected at cryogenic temperature, and the crystals were protected by soaking for a few minutes in mother liquor supplemented with $20 \%$ glycerol, before being flashfrozen in liquid nitrogen. Indexing and data reduction were performed using XDS [12], for data collection statistics see Table 1.

\section{Structure Determination, Model Building, and Refinement}

The structure in space group C222, was solved employing molecular replacement in MOLREP [13] using the high-resolution model of MtR2lox (PDB ID code 3EE4) [1]. A monomer was used as search model when positioning the four molecules in the asymmetric unit. Refinement by the maximum likelihood method was carried out in Refmac5 [14] including medium non-crystallographic 
symmetry (NCS) restrains between the four monomers. Manual inspection of electron density and building of the C-terminal helix was performed in COOT [15], where water molecules also were added and checked. The final model was validated using PROCHECK [16], RAMPAGE [17] and COOT. Geometry and refinement statistics are presented in Table 2. Coordinates and structure factors of $M t \mathrm{R} 2$ lox with an ordered C-terminal helix have been deposited at the Protein Data Bank with accession code 4AC8.

From the anomalous data sets, collected from a crystal of space group $\mathrm{P} 22_{1} 2_{1} 2_{1}$, model phased anomalous difference Fourier (DANO) maps were calculated using FFT in the CCP4 program suite [18].

All figures portraying protein models were prepared using PyMOL (www.pymol.org), and the Adaptive Poisson-Boltzmann Solver (APBS) plugin [19] was used for calculating electrostatic potentials.

\section{Results}

The new structures of MtR2lox show an ordered C-terminal helix

Here we present two new crystal forms of $M t \mathrm{R} 2 \mathrm{lox}$, the $\mathrm{C} 222_{1}$ crystal form containing two biological dimers in the asymmetric unit (Fig. 1A) and the $\mathrm{P} 22_{1} 2_{1}$ crystal form containing one biological dimer in the asymmetric unit, but with the same dimer-dimer interface as in $\mathrm{C} 222_{1}$ generated by a crystallographic two-fold symmetry. Interestingly, clear density for the previously disordered Cterminus was found in this new dimer-dimer interaction, and a 20 residue long alpha helix was built into the density (Fig. 1B). The helix, comprised of residues 
292-312, is positioned on the opposite side of the biological dimer interface and is one of the components, together with its symmetry equivalent, in a four-helix bundle that forms the interaction between the dimers in the crystal packing (Fig. $1 A, C)$.

The overall structure in $\mathrm{C} 222_{1}$ is highly similar to that observed in other crystal forms, and superposition of the monomer with the previously published structure (PDB ID code 3EE4) results in a root mean square deviation of $0.42 \AA$ over 282 $\mathrm{C}_{\mathrm{a}}$ atoms. Active site features, including the heterodinuclear Fe-Mn cofactor, the unique cross-link between Val-71 and Tyr-162 and the ligand occupying the ligand-binding cavity [1], are all conserved in the new structure. Anomalous data collected on the $\mathrm{P} 2{ }_{1} 2_{1} 2_{1}$ crystal form (Table 1 ) confirm the identity and position of $\mathrm{Mn}$ and $\mathrm{Fe}$ in the heterodinuclear cofactor.

Given the apparent ability of the C-terminal segment to adopt both an ordered and a disordered state we investigated what features bioinformatic predictors assign to the segment. The DisEMBL intrinsic protein disorder predictor (http://dis.embl.de/) [20] predicts no clear disorder tendency for the segment. IUPred (http://iupred.enzim.hu/) $[21,22]$ suggests that the C-terminal segment has the highest disorder tendency of the protein sequence, bordering on what would be predicted to be disordered. The Protein Structure Prediction Server (PSIPRED) [23] secondary structure predictor predicts the C-terminal sequence of $M t \mathrm{R} 2$ lox to be helical. Though a limited set of bioinformatics tools, the predictions agree well with the structural data presented here. Still, the disorder tendency would not be convincingly established using these tools. 
A new external metal-binding site in the crystal contact between the dimers

The dimer-dimer interface in the asymmetric unit contains a new external metalsite, where a calcium ion has been modeled and refined at full occupancy (Fig. $1 A, C)$. Anomalous data confirm that neither manganese nor iron is bound in this external site, and the high calcium content in the crystallization condition $(0.18$ M) makes it the most plausible metal in the site.

Coordination of the calcium ion is provided from both dimers, involving one carboxyl side chain from each of the four helices in the interface bundle. The interacting residues are Glu-304, located in the C-terminal helix, and Asp-266, from both monomers (Fig. 1C). Two solvent molecules also appear in the interaction, but the limited resolution of the structure prevents a detailed analysis of their interactions.

\section{Organization of the C-terminal helix shields a large positive surface patch}

Analysis of the surface potential of the previously published MtR2lox structure (spacegroup $\mathrm{P} 32$ 21, residues 291-314 disordered) reveals a pronounced concave positively charged patch on the surface of the monomer (Fig. 2A). In the present structure, the ordered C-terminal helix folds directly over the patch and shields the positive charge (Fig. 2B,C). The interface between the C-terminal helix, which contains several residues with negatively charged side chains, and the rest 
of the $M t \mathrm{R} 2$ lox monomer, is formed mainly by salt bridges between the helix and positively charged arginines in the patch (Fig. 3).

Alignment of R2lox sequences [2] indicate that the positive patch is likely conserved among the family, while the C-terminal helix is only highly conserved among the Mycobacterium genus and in a few strains of some Rhodococcus species. In other organisms the C-terminal segment varies in both sequence and length. It is however interesting to note that all sequences that contain a conserved C-terminal helix also have the key positive patch residues conserved. Examination of ribonucleotide reductase $\mathrm{R} 2$ protein structures and sequences further show that the positively charged patch is not a feature of $\mathrm{R} 2$ proteins.

\section{Discussion}

Here we show that the $\mathrm{C}$-terminal segment of the $M$. tuberculosis $\mathrm{R} 2 \mathrm{lox}$ can assume an ordered helical state to shield an evident positively charged concave surface of the protein. In these crystal forms, the C-terminal helix is part of a crystal contact mediated via a metal ion from the crystallization solution. Given the length of the segment, its helix propensity in secondary structure predictors, and the extensive interactions with the protein surface, we do not believe the structure and ordering of this segment to be a crystallization artifact. It appears likely, however, that the metal coordination and crystal contact provides further stabilization and allowed crystallographic trapping of the ordered state.

There is no structural, or other, support that the inter-dimer metal site is of biological relevance, and the metal ion is most likely simply mediating a crystal 
contact. This contact also produces an infinite chain of protein dimers in the crystal lattice, a very unlikely physiological arrangement. The concentration of calcium in the crystallization condition is further several orders of magnitude above physiological conditions.

It is now accepted that protein structure-function relationships are much more elaborate than the traditional view that function is associated with a single structured state. Structural dynamics in proteins occur from picoseconds up to milliseconds and are intricately coupled to several levels of function, from enzymatic chemical reactions to control of protein-protein interactions. A large portion of genomes also code for intrinsically unstructured proteins, or parts of proteins, a feature directly responsible for function in many cases [24-26]. In the well-studied ribonucleotide reductase $\mathrm{R} 2$ proteins the $\mathrm{C}$-terminal segment is disordered, but becomes ordered upon binding to protein R1, and is essential for proper radical transfer between the subunits [5]. R2lox and R2 proteins are sequence homologs and likely share ancestry. In this context it is interesting to note that, despite the complete change in function, the dynamic properties of the C-terminal segment appears conserved. For the R2lox proteins the detailed aspects of the function remain speculations at this time but we hypothesize that it is involved in shielding the positively charged patch, preventing unwanted interactions, until correct conditions for function are established, for example interaction with the biological membrane or a substrate-carrying protein. 


\section{Acknowledgements}

We acknowledge the access of beamtime at the European Synchrotron Research Facility, Grenoble, France. This work was supported by the Swedish Research Council (2010-5061), the Swedish Foundation for Strategic Research and the Knut and Alice Wallenberg Foundation.

\section{References}

[1] C.S. Andersson, M. Högbom, Proc. Natl. Acad. Sci. U.S.A., 2009, 106(14): p. 5633-5638.

[2] M. Högbom, J Biol Inorg Chem, 2010, 15(3): p. 339-349.

[3] M. Högbom, Metallomics, 2011, 3(2): p. 110-120.

[4] J.A. Cotruvo, J. Stubbe, Annu. Rev. Biochem., 2011, 80: p. 733-767.

[5] P. Nordlund, P. Reichard, Annu. Rev. Biochem., 2006, 75: p. 681-706.

[6] C.S. Andersson, M. Ohrstrom, A. Popovic-Bijelic, A. Gräslund, P. Stenmark, M. Högbom, J. Am. Chem. Soc., 2011 Dec 8 [Epub ahead of print] DOI: 10.1021/ja209678x

[7] O. Dym, E.A. Pratt, C. Ho, D. Eisenberg, Proc. Natl. Acad. Sci. U.S.A., 2000, 97(17): p. 9413-9418.

[8] N. Ben-Tal, B. Honig, R.M. Peitzsch, G. Denisov, S. McLaughlin, Biophys. J., 1996, 71(2): p. 561-575.

[9] S.A. Lloyd, F.G. Whitby, D.F. Blair, C.P. Hill, Nature, 1999, 400(6743): p. $472-475$. 
[10] T. Stams, Y. Chen, P.A. Boriack-Sjodin, J.D. Hurt, J. Liao, J.A. May, T. Dean, P. Laipis, D.N. Silverman, D.W. Christianson, Protein Sci., 1998, 7(3): p. 556-563.

[11] J.E. Guy, E. Whittle, M. Moche, J. Lengqvist, Y. Lindqvist, J. Shanklin, Proc. Natl. Acad. Sci. U.S.A., 2011, 108(40): p. 16594-16599.

[12] W. Kabsch, Acta crystallogr., Sect. D, 2010, 66(Pt 2): p. 125-132.

[13] A. Vagin, A. Teplyakov, J. Appl. Crystallogr., 1997, 30: p. 1022-1025.

[14] G.N. Murshudov, A.A. Vagin, E.J. Dodson, Acta Crystallogr., Sect. D, 1997, 53(Pt 3): p. 240-255.

[15] P. Emsley, K. Cowtan, Acta Crystallogr., Sect. D, 2004, 60: p. 21262132.

[16] R.A. Laskowski, M.W. MacArthur, D.S. Moss, J.M. Thornton, J. Appl. Crystallogr., 1993, 26: p. 283-291.

[17] S.C. Lovell, I.W. Davis, W.B. Arendall, 3rd, P.I. de Bakker, J.M. Word, M.G. Prisant, J.S. Richardson, D.C. Richardson, Proteins, 2003, 50(3): p. 437-450.

[18] N. Collaborative Computational Project, Acta Crystallogr., Sect. D, 1994, 50(Pt 5): p. 760-763.

[19] N.A. Baker, D. Sept, S. Joseph, M.J. Holst, J.A. McCammon, Proc. Natl. Acad. Sci. U.S.A., 2001, 98(18): p. 10037-10041.

[20] R. Linding, L.J. Jensen, F. Diella, P. Bork, T.J. Gibson, R.B. Russell, Structure, 2003, 11(11): p. 1453-1459.

[21] Z. Dosztanyi, V. Csizmok, P. Tompa, I. Simon, Bioinformatics, 2005, 21(16): p. 3433-3434. 
[22] Z. Dosztanyi, V. Csizmok, P. Tompa, I. Simon, J. Mol. Biol., 2005, 347(4): p. 827-839.

[23] L.J. McGuffin, K. Bryson, D.T. Jones, Bioinformatics, 2000, 16(4): p. 404-405.

[24] Z. Bu, D.J. Callaway, Adv. Protein Chem. Struct. Biol., 2011, 83: p. 163221.

[25] R.M. Daniel, R.V. Dunn, J.L. Finney, J.C. Smith, Annu. Rev. Biophys. Biomol. Struct., 2003, 32: p. 69-92.

[26] H.J. Dyson, P.E. Wright, Nat. Rev. Mol. Cell. Biol., 2005, 6(3): p. $197-$ 208. 
Table 1. Data collection statistics. Values in parenthesis represent the highest resolution shell.

\begin{tabular}{|l|c|c|c|}
\hline & C222 & \multicolumn{2}{|c|}{ P2 $2_{1} \mathbf{2}_{1}$} \\
\hline Wavelength $(\AA)$ & 1.127 & 1.7 & 1.8 \\
\hline Cell dimensions : & & & \\
\hline \multicolumn{1}{|c|}{$a, b, c(\AA)$} & $110.0,210.9,140.0$ & $43.6,119.1,136.0$ & $43.6,119.1,136.0$ \\
\hline Resolution $(\AA)$ & $36.7-2.75(2.83-2.75)$ & $50-3.1(3.26-3.07)$ & $50-2.9(3.07-2.89)$ \\
\hline$R_{\text {sym }}(\%)$ & $14.4(61.1)$ & $11.4(68.5)$ & $9.6(52.1)$ \\
\hline Mn(I/s(I)) & $9.75(2.83)$ & $11.44(2.48)$ & $12.25(2.61)$ \\
\hline Completeness $(\%)$ & $92.1(94.4)$ & $96.5(91.4)$ & $96.3(91.5)$ \\
\hline Redundancy & $5.6(5.5)$ & $3.5(3.4)$ & $3.8(3.8)$ \\
\hline SigAno & - & $0.90(0.72)$ & $0.92(0.76)$ \\
\hline
\end{tabular}


Table 2. Refinement statistics for the native data set in space group C222

\begin{tabular}{|c|c|}
\hline Refinement program & Refmac 5.6.0117 \\
\hline Resolution $(\AA)$ & $36.72-2.75$ \\
\hline $\begin{array}{l}\text { No. unique reflections } \\
\text { working / test }\end{array}$ & $37284 / 1963$ \\
\hline$R_{\text {work }} / R_{\text {free }}(\%)$ & $22.0 / 26.8$ \\
\hline Monomers / AU & 4 \\
\hline No. atoms & \\
\hline Protein & 9939 \\
\hline Metal ions & 10 \\
\hline Ligands & 70 \\
\hline Solvent & 88 \\
\hline$B$-factors $\left(\AA^{2}\right)$ & \\
\hline Protein & 31.7 \\
\hline Metal ions & 28.0 \\
\hline Ligands & 33.1 \\
\hline Solvent & 22.8 \\
\hline R.m.s. deviations & \\
\hline Bond lengths $(\AA)$ & 0.007 \\
\hline Bond angles $\left({ }^{\circ}\right)$ & 1.12 \\
\hline $\begin{array}{l}\text { Ramachandran distribution: } \\
\text { Favoured / Allowed (\%) }\end{array}$ & $97.7 / 2.3$ \\
\hline PDB accession code & $4 \mathrm{AC} 8$ \\
\hline
\end{tabular}




\section{Figures}

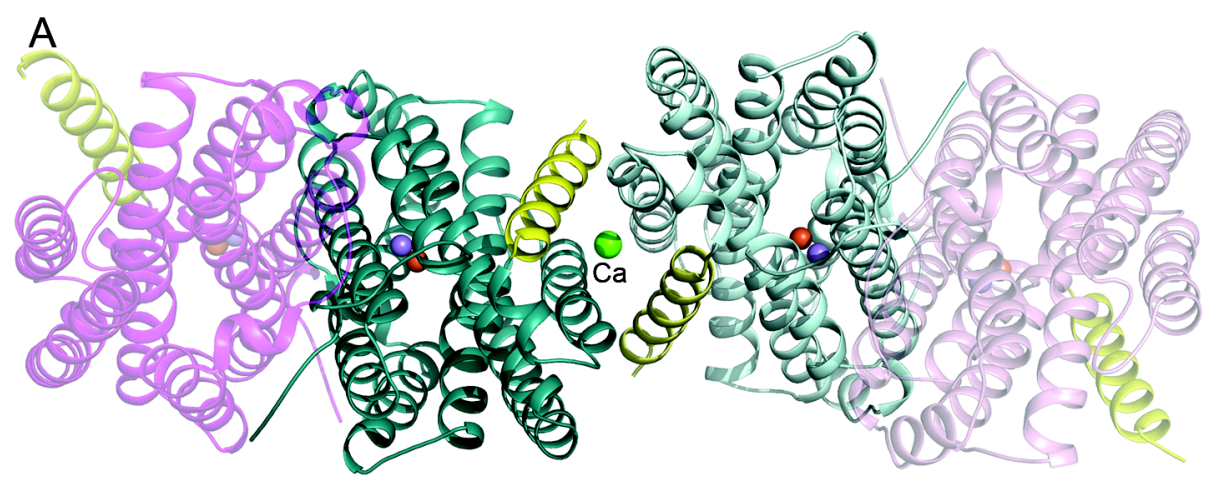

B

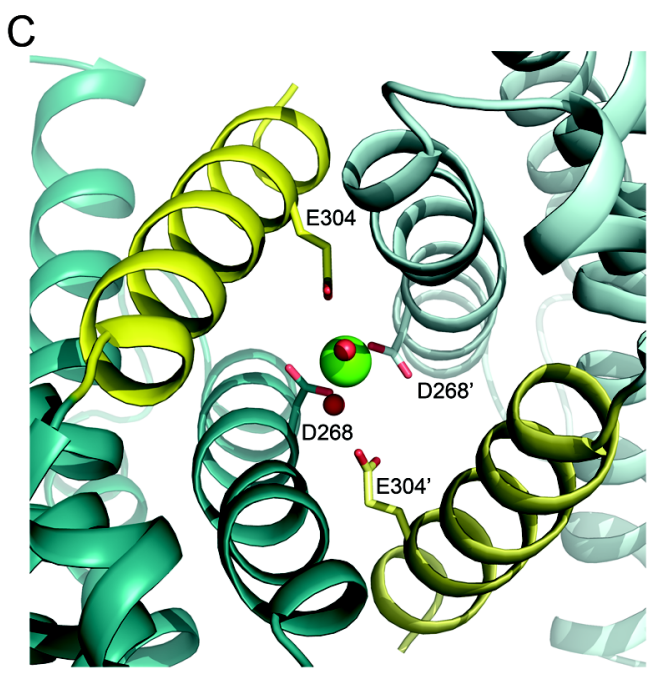

Fig. 1

A. Crystal packing of the two biological dimers with the ordered C-terminal helix shown in yellow. The interface between the biological dimers contains a new external metal site with a calcium ion bound. Each dimer contributes with two helices (where one is the C-terminal helix) to the symmetrical helical bundle forming the site. Metal ions are indicated as spheres, with calcium in green, manganese in purple and iron in orange.

B. Electron density for the C-terminal helix. Fo-Fc omit map contoured at 0.12 $\mathrm{e} \AA^{-3}$. 
C. Close-up view of the external metal site. Residue Glu-304 and Asp-268 from both monomers coordinate the calcium. Red spheres show two solvent molecules. Residues labeled with a prime are from the second biological dimer.
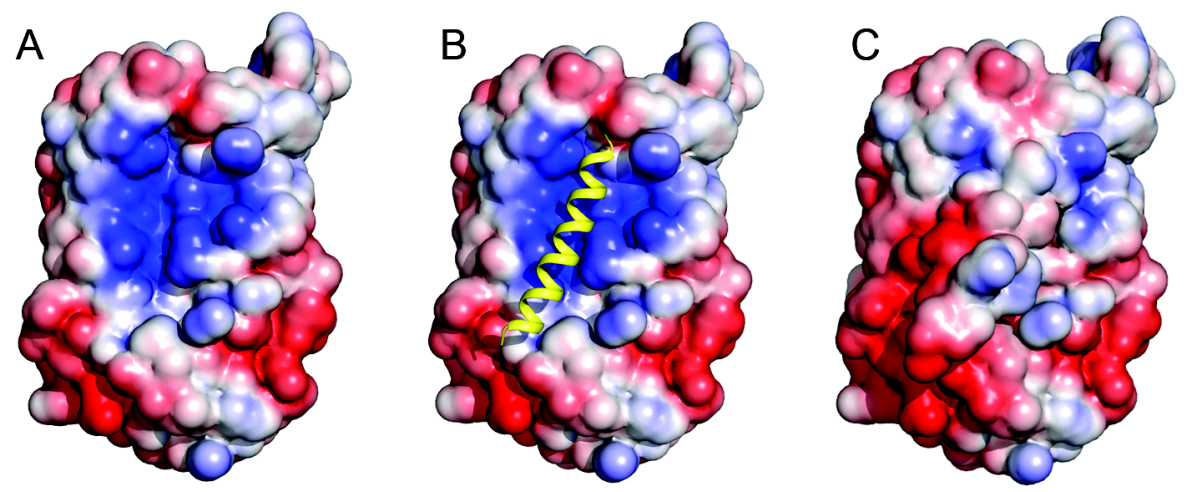

Fig. 2

Overall surface contact potential of the $M t \mathrm{R} 2 \mathrm{lox}$. Red and blue indicate negative and positive surface contact potential, respectively. Panel A shows the surface contact potential of the previously published $M t$ R2lox structure (PDB ID: 3EE4) [1] with a disordered C-terminus, displaying a distinct concave positive patch. Panel B visualizes the position of the ordered C-terminal helix in the new structures presented here, panel $\mathrm{C}$ shows the surface potential calculated for the structure including the ordered C-terminal helix. 


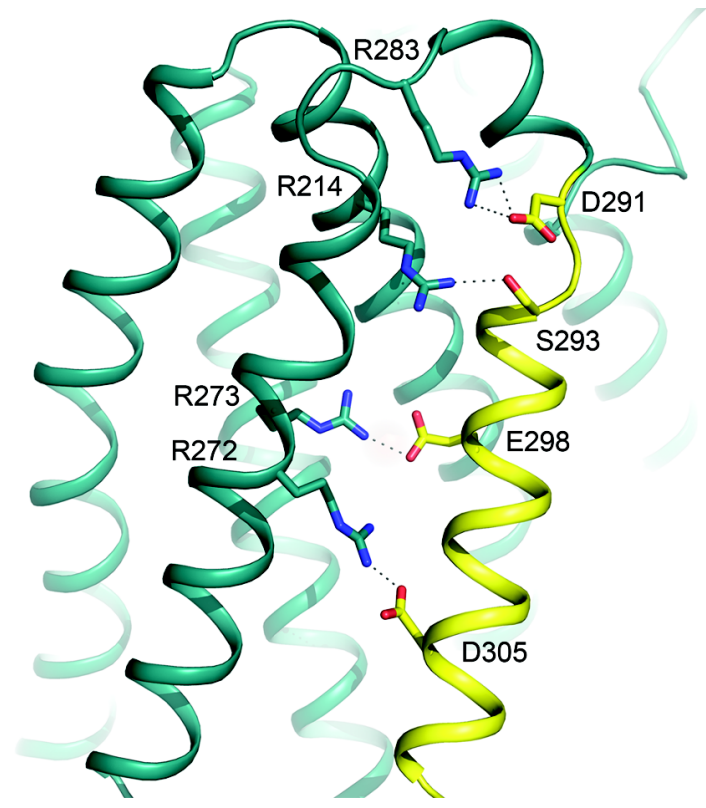

Fig. 3

Close-up of the interactions between the ordered C-terminal helix and the positively charged surface patch. Dashed lines indicate hydrogen bonds and charge-charge interactions. 